\title{
Me dijeron que soy crónico: lo que estoy haciendo para cuidarme
}

\author{
Fabiola Castellanos Soriano²
}

Daniel Eslava Albarracín ${ }^{3}$

doi:10.11144/Javeriana.IE16-1.mdsc

Cómo citar: Castellanos Soriano F, Eslava Albarracín D. Me dijeron que soy crónico: 1o que estoy haciendo para cuidarme. Investig Enferm. Imagen Desarr. 2014;16(1):11-26. doi:10.11144/Javeriana.IE16-1.mdsc

1. Artículo de investigación. Recibido: 29 de enero de 2014. Revisado: 5 de febrero de 2014. Aceptado: 10 de febrero de 2014. Proyecto financiado por la Oficina de Fomento a la Investigación, Vicerrectoría Académica, Pontificia Universidad Javeriana. Código: 12010 U40401200.

2. Enfermera. Doctora en Enfermería, Universidad Nacional de Colombia, Bogotá, Colombia. Directora, Departamento de Enfermería en Salud de los Colectivos. Docente, Facultad de Enfermería, Pontificia Universidad Javeriana, Bogotá, Colombia. Líder del grupo de investigación Conceptualización y Práctica de Enfermería. Correo electrónico: fabiola. castellano@javeriana.edu.co.

3. Enfermero. Doctor en Salud Pública. Director de la Oficina de Posgrados. Docente, Facultad de Enfermeria, Pontificia Universidad Javeriana, Bogotá, Colombia. Líder del grupo de investigación procesos Sociales y Salud. Correo electrónico: dgeslava@javeriana.edu.co. 


\section{Resumen}

Objetivo: Describir la trayectoria de cuidado popular en un grupo de personas mayores con enfermedades crónicas que vive en una zona urbano-marginal de la ciudad de Bogotá, Colombia. Materiales y métodos: En el estudio cualitativo de tipo etnográfico se utilizó la observación participante y entrevistas semiestructuradas. El análisis de datos fue manual y siguió las fases propuestas por Leininger. Resultados: De las 18 personas mayores entrevistadas emergieron tres patrones culturales: 1) soy declarado enfermo crónico, 2) como soy enfermo crónico busco ayuda de los que conocen la enfermedad, 3) los enfermos crónicos cuidamos el cuerpo de muchas maneras. Conclusiones: La trayectoria de cuidado popular revela diversas formas de cuidarse. Inicia con la construcción de significados sobre la enfermedad crónica, luego pasa a la toma de decisiones (donde la familia interviene de forma rápida en momentos de crisis de la enfermedad) y, por último, se establecen cuidados populares, que son los más llamativos el dejar descansar el cuerpo

Palabras clave: enfermedad crónica, investigación cualitativa, personas mayores, enfermeria en salud comunitaria, enfermería transcultural.

Palabras clave descriptores: enfermedad crónica, investigación cualitativa, ancianos, enfermería en salud comunitaria, enfermería transcultural

\section{I was told I 'm chronic: what I'm doing to take care of myself}

\section{Abstract}

Objective: To describe the trajectory of popular care in a group of older people with chronic diseases living in a marginal urban area of the city of Bogotá, Colombia. Materials and Methods: Qualitative ethnographic study which included participant observation and semi-structured interviews. The data analysis was manual and followed the phases proposed by Leininger. Results: Of the 18 senior people interviewed three cultural patterns emerged: 1) I am declared chronically ill, 2) as I am chronically ill, I seek help from those who know about the disease, 3) the chronically ill take care of the body in many ways. Conclusions: The trajectory of popular care reveals various forms of care. It begins with the construction of meanings about chronic disease, then proceeds to decision making (where the family is quickly involved in moments of crisis of the disease) and, finally, popular care, being the most striking letting the body rest.

Keywords: chronic illness, qualitative research, senior people, community health nursing, transcultural nursing

Keywords plus: chronic disease, qualitative research, aged, community health nursing, transcultural nursing 


\section{Disseram-me que estou crônico: o que estou fazendo para me cuidar}

\section{Resumo}

Objetivo: Descrever a trajetória de cuidado popular em uma turma de idosos com doenças crônicas, moradores de uma zona urbano-marginal da cidade de Bogotá, Colômbia. Materiais e métodos: No estudo qualitativo de tipo etnográfico utilizou-se a observação participante e entrevistas semiestruturadas. A análise de dados foi manual e seguiu os estágios propostos por Leininger. Resultados: Dos 18 idosos entrevistados emergiram três padrões culturais: 1) a gente foi declarada doente crônica, 2) como a gente está doente crônica vai procurar ajuda dos que conhecem da doença, 3) os doentes crônicos cuidamos do corpo de muitas maneiras. Conclusões: A trajetória de cuidado popular desvenda diversas formas de cuidar. Inicia com a construção de significados sobre a doença crónica para passar à tomada de decisões (onde a família intervém de forma rápida em momentos de crise da doença) e, por fim, estabelecem-se cuidados populares, onde remarca-se deixar o corpo descansar.

Palavras chave: doença crônica, pesquisa qualitativa, idosos, enfermagem em saúde comunitária, enfermagem transcultural

Palavras chave descriptor: doença crônica, pesquisa qualitativa, idoso, enfermagem em saúde comunitária, enfermagem transcultural 


\section{Introducción}

La proporción de personas mayores de 60 años de edad está aumentando más rápidamente que cualquier otro grupo de edad en casi todos los países (1). Esta situación enfrenta a los profesionales de la salud a nuevos desafíos en el cuidado de las personas mayores de diversas culturas. Algunos desafios están relacionados con la formación del personal de salud para la atención de las personas mayores con enfermedades crónicas, la creación de políticas sostenibles para la atención paliativa de larga duración y el diseño de servicios y entornos adaptados a estas personas (2).

Las enfermedades no transmisibles, también conocidas como enfermedades crónicas (EC), son de larga duración y, por lo general, evolucionan lentamente. Estas enfermedades son la principal causa de muerte y discapacidad de personas mayores para ambos sexos. En el mundo, en 2008, de los 57 millones de muertes, 36 millones (63\%) se debieron a enfermedades no transmisibles, especialmente a enfermedades cardiovasculares, diabetes, cáncer y enfermedades respiratorias crónicas (3). En Colombia, las cuatro principales EC son la enfermedad isquémica del corazón, las enfermedades respiratorias crónicas, la diabetes y el cáncer (4).

La EC trae consigo malestares y complicaciones y promueve el uso frecuente de los servicios de salud. Por ende, corresponde a la sociedad participar de manera activa, democrática y equitativa en la puesta en marcha de los servicios que se ofrecen para garantizar la atención necesaria de las personas mayores que viven con EC (5).

La atención competente en salud requiere que los profesionales de la salud comprendan que las personas tienen sus propias construcciones sobre la salud y la enfermedad, las cuales son producto de la cultura en la que viven y de sus experiencias en el cuidado de salud (6).

Por ello, buscando esta comprensión sobre el cuidado de la salud según la cultura, Castellanos (7) propone la trayectoria de cuidado popular de los ancianos como una alternativa que permite conocer la manera como cuidan su salud. La autora conceptualiza dicha trayectoria como el proceso que siguen los ancianos para establecer su cuidado, en respuesta a diversas situaciones. Esta trayectoria posee tres componentes que se articulan secuencialmente: comprensión de la situación de salud actual, toma de decisiones y ejecución del cuidado (7).

Reconocer esa trayectoria de cuidado popular de las personas mayores con enfermedades crónicas puede ayudar a los profesionales de salud a comprender, actuar y dar cuidado de forma competente desde la perspectiva cultural.

En el contexto expuesto, y frente a la necesidad inaplazable de brindar cuidado culturalmente congruente, este estudio se orienta a describir la trayectoria de cuidado popular en un grupo de personas mayores con EC, que vive en una zona urbano-marginal de la ciudad de Bogotá, Colombia.

Las trayectorias de cuidado popular de las EC no son similares; varían según su tipo. A pesar de esto, es posible identificar diferencias y similitudes en el cuidado (8). En este documento se presentan las similitudes de la trayectoria de cuidado popular de las personas mayores con hipertensión 
arterial (HTA), diabetes mellitus tipo II y enfermedad pulmonar obstructiva crónica (EPOC). Las diferencias en las trayectorias de cuidado del grupo de personas mayores colaboradoras serán publicadas en otro documento.

\section{Metodología}

Este estudio cualitativo de tipo etnográfico se desarrolló en la ciudad de Bogotá, en barrios urbano-marginales de las localidades de Chapinero y Usme. Se utilizó la etnografía porque permite adentrarse y comprender de forma amplia las trayectorias de cuidado popular de personas mayores con enfermedades crónicas, explorando la subjetividad de los sujetos y el contexto.

La selección de los participantes fue intencional, porque en la investigación cualitativa lo que interesa es la profundidad sobre la extensión (9), y se tiene en cuenta el criterio de saturación teórica. Además, no se puede establecer el número de colaboradores a priori, ya que antes de un contacto efectivo con el campo, en la medida en que el investigador se interna en la dinámica, se conocen los discursos, las prácticas y los grupos relevantes y significativos para la descripción (10).

Algunos colaboradores se seleccionaron de los programas de asistencia social, otros pertenecían a los programas de atención en salud para personas con enfermedades crónicas, y otros, que no asistían a ningún programa de prevención o de asistencia social, se captaron por recomendación de las personas participantes en los barrios de las localidades mencionadas, gracias a la técnica de bola de nieve. Estos colaboradores tenían enfermedades crónicas confirmadas por diagnóstico médico y participaron de forma voluntaria.

La información se recolectó por medio de observación participante en las actividades de los grupos de asistencia social y en actividades cotidianas (preparación del desayuno y aseo de la casa) en los hogares de los colaboradores. Las entrevistas se desarrollaron en salones comunales o en las viviendas de los colaboradores, y duraron entre treinta minutos y dos horas.

La investigadora principal y profesionales de salud entrenados 1levaron a cabo las entrevistas. Estas últimas se captaron con una grabadora digital de audio y se transcribieron literalmente en un procesador de texto.

La información derivada de la observación participante y de las entrevistas se revisó y analizó siguiendo a Wolcott (11). También se tuvo en cuenta a Leininger (8), quien propone cuatro fases de análisis: en la fase 1 , de recolección y análisis de la información, se generaron guías para las entrevistas con los colaboradores y para la descripción del contexto.

Para las fases 2, identificación y categorización de descriptores y componentes, y 3, generación de patrones y análisis contextual, se elaboraron varias matrices para cada colaborador, que incluian descriptores, categorias iniciales y análisis del investigador. Estas matrices se revisaron en varias ocasiones para precisar las categorías y generar los patrones culturales que describían cada elemento de la trayectoria de cuidado popular de las personas mayores con enfermedades crónicas. Se contó con la participación de otros observadores y expertos en los momentos de análisis de la información, a fin de mantener la credibilidad de los datos. 
En la última fase, de generación de temas mayores, se construyó un tema mayor resultado de la comprensión de la información, que involucra los tres elementos de la trayectoria de cuidado popular de las personas mayores con EC: comprensión de la situación de salud, toma de decisiones y ejecución del cuidado. Esta fase 4 es la más alta del análisis de datos, porque requiere síntesis de pensamiento, configuraciones, análisis e interpretación de los resultados a la luz de los objetivos y formulaciones creativas de los datos de las fases previas (8).

Los resultados se complementaron con diálogos de los colaboradores como evidencia de los aspectos planteados por la investigadora en la interpretación. Por consideraciones éticas, se mantuvo la confidencialidad de la información de los colaboradores y de los participantes. A las entrevistas se les asignaron dos letras y un número para su identificación. Todos los colaboradores firmaron el consentimiento informado. Esta investigación inició en marzo de 2011 y finalizó en diciembre de 2012, la cual contó con el aval del Comité de Ética e Investigación de la Pontificia Universidad Javeriana.

\section{Resultados}

\section{Características de las personas mayores}

Los participantes fueron 18 personas mayores, 13 mujeres y 5 hombres en edades entre los 56 y 90 años. Tenían diagnósticos de HTA (7 personas), EPOC (6 personas) y diabetes (5 personas).

\section{Patrones culturales}

Los tres patrones culturales que se desarrollan a continuación conforman el tema mayor que da nombre a este artículo: 1) soy declarado enfermo crónico, 2) como soy enfermo crónico busco ayuda de los que conocen la enfermedad y 3) los enfermos crónicos cuidamos el cuerpo de muchas maneras.

\section{Soy declarado enfermo crónico}

Este patrón revela la comprensión de la enfermedad crónica como primer elemento de la trayectoria de cuidado popular de las personas mayores con enfermedades crónicas. A continuación, se describen las cuatro categorías que componen este patrón:

a) Siento maluqueras y el médico dijo que era crónico: esta categoría responde a la descripción de los signos y los síntomas en el cuerpo de la persona. La identificación inicial de la EC por la persona mayor es muy difusa, debido a que los síntomas y los signos son asociados a enfermedades agudas. 
La persona mayor percibe un deterioro progresivo y ubica la enfermedad en partes de su cuerpo. Por ejemplo, para la EPOC, los pulmones son el centro de la enfermedad; en la diabetes se ubica en la sangre; en el caso de la hipertensión está en la cabeza o en la sangre. El cuerpo es visto como una máquina a la cual se le dañan algunas partes y deja de funcionar adecuadamente.

La EC se detecta en el momento de una crisis o después de la atención médica y con los resultados de los exámenes clínicos. El médico le da nombre a la enfermedad y suministra recomendaciones para su tratamiento y recuperación. Así la enfermedad empieza a existir en la vida de la persona mayor bajo el rótulo dado por el médico y la persona empieza a darle sentido a lo que sucede en su cuerpo:

Yo vivía con malestares, hasta que me ¡desmayé! Me llevaron al médico y con los exámenes me dijeron que era diabética... Desde que me descubrieron la diabetes, hay veces que uno se siente mareado, con pereza, mucho sueño, le da mucha sed, y a uno se le va mucho la visión. (CC, entrevista 1$)$

b) El malestar da por temporadas, aunque a veces uno se alienta: uno de los principales hallazgos de este estudio es el hecho de que los colaboradores no entienden el concepto técnico de EC. Tienen una noción de lo crónico como "algo que no se cura" o algo "terminal"; sin embargo, cuando explican sus propias enfermedades crónicas, creen que pueden aliviarse.

Una concepción frecuente en el grupo de colaboradores es creer que no están enfermos, porque no presentan los signos o los síntomas de la enfermedad. Es decir, se percibe la recuperación de la salud cuando la enfermedad se estabiliza debido al tratamiento.

Los colaboradores comparan la EC con la enfermedad infecciosa, que en muchas ocasiones se cura después del tratamiento. También suelen confundirse las complicaciones con los signos comunes de la enfermedad:

Cuando me descubrieron la diabetes fue terrible para mí, porque mi mamá también tenía diabetes y sufrió mucho; duró hartísimo tiempo con insulina. En este momento es ancianita, ya le pasó la diabetes, y no le ponemos insulina. (CC, entrevista 1$)$

c) Ahora las enfermedades tienen nombre: esta categoria expresa la comprensión que tienen los colaboradores sobre el conocimiento de las enfermedades. La transferencia de la información de los servicios de salud familiariza a los colaboradores con algunos conceptos sobre las enfermedades, en especial las infecciosas. Sin embargo, los nombres y las explicaciones sobre el origen de las EC son nuevos. No es claro para ellos el curso de la enfermedad, ni sus consecuencias. Aspectos que dan sentido a la falta de claridad del término enfermedad crónica que, además, es un concepto originado desde la técnica y no desde el conocimiento popular, como sî lo es el término dado a algunas enfermedades respiratorias como la gripa, por ejemplo. 
A pesar de esto, y para dar sentido a su situación, ellos construyen sus propias nominaciones para estas enfermedades; por ejemplo, la EPOC es lo mismo que la bronquitis, la llaman también ahogo, gripa, enfermedad de la tos o ataque a los pulmones: "Donde yo nací la gente se moría y nunca decían se murió de diabetes, o de otra cosa, nada... se murió y ¡listo! Las enfermedades se están conociendo en esta época, les ponen nombre ahorita" (CC entrevista 1$)$.

d) Creo que muchas cosas me enfermaron: para las tres enfermedades estudiadas (EPOC, diabetes e HTA) las atribuciones causales están centradas en la dimensión física y en la socioeconómica. El origen de la enfermedad es externo al individuo, dado por la naturaleza o el ambiente (frío), por la dieta o como consecuencia de agentes relacionados con el trabajo. Otros son internos o del individuo, como la herencia. Estas atribuciones causales están influenciadas por los servicios de salud y, en ocasiones, entran en conflicto con las explicaciones propias.

Además, estos colaboradores han establecido una jerarquización de las enfermedades, teniendo en cuenta su cercanía con la muerte. El cáncer y la diabetes son las enfermedades más graves, seguidos por la EPOC y la HTA:

Una enfermedad crónica como el cáncer, de eso uno no se alienta, ¡nunca! Ahí sí con Dios y la muerte... Eso sí es crónico. Y la diabetes es crónica, porque las personas no pueden comer de todo y lo que comen no les alimenta, entonces ahí está lo grave. (E entrevista 2)

\section{Como soy enfermo crónico busco ayuda de los que conocen la enfermedad}

Todos los elementos que constituyen la comprensión de la EC se conjugan y orientan a la persona para la toma de decisiones de cuidado, que es el segundo elemento de la trayectoria de cuidado popular de las personas mayores con EC. La toma de decisiones en este estudio tiene dos escenarios: en el primero se busca la atención cuando se presentan crisis de la enfermedad. En el segundo escenario se asiste a los controles periódicos en los servicios de salud. A continuación, se describen las dos categorías que componen este patrón:

a) Yo aguanto las maluqueras, pero a veces me llevan al hospital: las personas mayores experimentan sintomas y signos frecuentes que no alteran la cotidianidad. Por ejemplo, la sed en la diabetes, la tos en la EPOC y el calor en la cara en la HTA. También sienten los síntomas de las complicaciones como extremo dolor, sensación de desmayo o pérdida de conocimiento. Sin embargo, este grupo de colaboradores tiene dificultad para diferenciar entre los síntomas importantes para consultar y los sintomas de las complicaciones. Ellos buscan ayuda cuando la complicación ya está presente. Adicionalmente, cuando existen varias condiciones crónicas de salud, priorizan la enfermedad que genera más limitaciones y que se considera más peligrosa. Esta enfermedad, por lo general, guía el cuidado. 
En esta condición de salud, el criterio de gravedad es aplicado para buscar los servicios de salud, porque hay limitación en las actividades de la vida diaria, temor a la complicación (un evento cerebrovascular, dificultad respiratoria o pérdida del conocimiento) y la cercanía de la muerte. Para la toma de decisiones, continúa siendo central la familia que en momentos de crisis toma el control de la situación.

No se acude a los servicios de salud de manera voluntaria cuando se trata de la hospitalización. Quedarse en un hospital determina la pérdida de autonomía y la cercanía con la muerte. El temor a la hospitalización es una de las razones para usar prácticas populares y retardar la búsqueda de atención médica:

Yo me aguanto muchos dolores. Yo aguanto en la casa porque no me gusta estar hospitalizada, me dan nervios... Pero cuando mi hijo o mis hijas se dan cuenta de mi dolor de cabeza, me llevan al hospital, yo no alcanzo a hablar cuando ya me quieren cargar para el hospital (se ríe). Ellos dicen: ¡Ay, no!, tiene que ir al médico, tiene que ir... (A: entrevista 1)

b) Yo voy a los controles para que el médico me revise: en el segundo escenario, la persona mayor decide asistir a controles en los servicios de salud, dada la influencia que tiene la explicación del personal de salud para la comprensión de la EC y el éxito de los tratamientos en el control de la enfermedad:

Después de que renuncié al trabajo quedé en manos del médico porque él me controlaba, me mandaba medicamentos, hasta que ya no hubo nada más que hacer sino el cateterismo. La operación salió muy bien gracias Dios, estoy muy recuperado... Creo que a mí me curaron porque no siento nada. (HM: entrevista 1)

\section{Los enfermos crónicos cuidamos el cuerpo de muchas maneras}

El tercer elemento de la trayectoria de cuidado popular es la ejecución del cuidado, que conjuga y hace tangibles los dos elementos previos: la comprensión de la situación de salud y la toma de decisiones. Un aspecto para resaltar es que el cuidado se inicia de manera consciente a partir del diagnóstico médico de la EC, es decir, la enfermedad existe como una entidad que está ubicada en un órgano o sistema del cuerpo; por lo tanto, las personas procuran cuidados para recuperar, mantener o proteger la salud (12).

Los momentos de crisis de la EC son enfrentados con cuidados dirigidos a recuperar la salud. Cuando la enfermedad se mantiene estable, es decir, no hay signos o sintomas evidentes que afecten de manera significativa la funcionalidad de la persona, se buscan cuidados para mantener la salud.

También se identificaron cuidados dirigidos a la protección de la salud, aunque en menor proporción. A su vez, todos estos cuidados populares 
se dirigen a diferentes dimensiones del ser: la física, la mental y la espiritual (12), siendo los cuidados de la dimensión física los más predominantes, dado que se atiende a las recomendaciones médicas integradas al propio concepto de salud.

Para la selección de los cuidados, se encontró que se accede inicialmente a los recursos propios: experiencia, información de la familia y los amigos; la consulta de médicos particulares, y luego se busca el cuidado en los servicios de salud. La presencia o ausencia de los síntomas de la EC determina que la práctica del cuidado sea intermitente o continua.

Las personas mayores con EC evalúan la efectividad de las prácticas cuando encuentran que sus sintomas mejoran. Este es un proceso dinámico y puede ser cíclico, es decir, a partir de la evaluación de la efectividad del cuidado pueden volver a consultar en sus propios recursos o acudir a los servicios de salud. El patrón refleja en tres categorías los tres propósitos de la ejecución del cuidado popular.

a) Para mantenernos bien, hay que hacer de todo un poco: en esta categoria, mantenerse bien significa buscar el equilibrio en las dimensiones física, mental y espiritual. Así, los cuidados para mantenerse bien están dirigidos en mayor proporción a la dimensión física, a efectos de controlar la aparición de los síntomas que pueden generar malestar.

Algunas prácticas de cuidado popular desde la dimensión física son: asistir a los controles, tomar los medicamentos, hacer ejercicio, seleccionar los alimentos que se pueden o no consumir, comer adecuadamente de acuerdo con sus posibilidades económicas y tomar aguas de hierbas. En la dimensión mental: realizar actividades que ocupen el tiempo, mantener la calma frente a las dificultades, compartir con la familia, hablar con los vecinos o no dejarse afectar por la enfermedad. Y en la dimensión espiritual: orar.

b) Entre Dios, los médicos y la familia, más aliviados nos sentimos: se refiere a las acciones para recuperar la salud en momentos de crisis de la EC. Sus síntomas se presentan exacerbados y el cuidado en las dimensiones física, mental y espiritual se hace continuo hasta la finalización de la crisis o hasta que se percibe que la vida ya no está en peligro.

Los colaboradores reconocen que los recursos propios tienen una utilidad limitada y en momentos de crisis deben buscar ayuda profesional, acudiendo al hospital por urgencias. Las recomendaciones de cuidado dadas en los servicios de salud son llevadas a cabo en el hogar; sin embargo, se pueden alterar o suspender por factores como la sensación de bienestar o la percepción de cura de la enfermedad (cuando desaparecen los síntomas y ya no interfieren de forma significativa en la vida de la persona). También la percepción de efectos secundarios negativos de los medicamentos sobre el cuerpo genera la creencia y la práctica de que el cuerpo se debe dejar descansar para evitar dañarlo. Las dificultades económicas también influyen en la continuidad de los tratamientos.

En la dimensión espiritual, las oraciones y la asistencia a los rituales religiosos son prácticas frecuentes. Esta dimensión se expresa con mayor fuerza en momentos en los cuales la vida está en peligro y esta se 
deja en manos de un ser supremo. Se observa en los colaboradores resignación ante su situación y se asume la enfermedad como parte del proceso de envejecimiento.

Algunas prácticas de cuidado popular desde la dimensión física son: utilizar ropa adecuada al clima o consumir frutas, plantas o ciertos alimentos. Seleccionar los medicamentos y tomarlos cuando lo consideren necesario. En lo mental: recibir y aceptar el apoyo familiar. En lo espiritual: orar por la salud, asistir a misas y dejar la vida en manos de Dios.

c) Evitamos lo que nos hace mal y confiamos en Dios: esta categoría presenta el propósito de proteger la salud. Los cuidados están dirigidos de forma consciente hacia las tres dimensiones del cuidado: físico, mental y espiritual. Evitar lo que hace mal significa tratar de evadir los riesgos del contexto ambiental o familiar y buscar el equilibrio. Para estos colaboradores proteger la salud en medio de una EC implica fomentar la autonomía y sentirse funcionales. Están más atentos a detectar y evitar los riesgos del entorno que pueden afectar la salud y poner en peligro la vida.

Algunas prácticas de cuidado físico son: evitar las fiestas, el licor y las caídas. Las de cuidado mental: evitar las peleas, los disgustos y las preocupaciones, y ser paciente ante las situaciones dificiles. Y las del cuidado espiritual: tener fe en Dios, en la Virgen y otros santos.

\section{Discusión}

Estos resultados se analizaron bajo la propuesta de Castellanos (7): el modelo de cuidado cultural en la adversidad, que contiene la trayectoria de cuidado (13), el modelo de tradiciones de salud de Spector (12) y la literatura, que permitieron identificar las similitudes de la trayectoria de cuidado de las EC analizadas.

Para este grupo de colaboradores, sus experiencias se remiten a enfermedades infecciosas, que son sustancialmente diferentes en su curso y tratamiento de las EC. Este hallazgo coincide con algunos estudios en los cuales se menciona que una cuarta parte de las personas no saben que tienen una EC (14). Machado y Car (15), en un estudio con personas que sufren de HTA, llegan a similares conclusiones respecto al conocimiento de las enfermedades agudas frente a las crónicas, lo cual puede generar abandono de medicamentos y tratamientos.

Además, estos autores en el mismo estudio, identificaron que la presencia de los síntomas y signos es sinónimo de la presencia de la enfermedad y la ausencia sinónimo de salud (15). Así, las sensaciones del cuerpo son las que determinan la existencia de la enfermedad y movilizan a la persona para dejar o continuar el tratamiento.

Para los colaboradores de este estudio, el origen de la enfermedad es externo (frío, dieta y trabajo) e interno (la herencia). De manera similar, algunos autores identifican que el trabajo, las preocupaciones y las sorpresas son causas de las enfermedades (16), al igual que la herencia y el nerviosismo, aun cuando en otros casos las personas no se consideran enfermas (15) o la enfermedad no es claramente identificada (17). 
En consecuencia, el no identificar de forma clara la EC y no tener en cuenta los riesgos que supone, lleva a las personas mayores de este estudio a tomar decisiones que motivan la asistencia en momentos de crisis de la enfermedad. Autores como Acosta Gonzáles y colaboradores (18) encontraron en su estudio que solo la mitad de los encuestados identificaron su condición de salud como una amenaza. En otros estudios se encuentra que la gravedad de la condición es el detonante para buscar ayuda $(7,19)$. El dolor agudo también es uno de los principales elementos que llevan a buscar ayuda (20), aunque esto puede variar de cultura en cultura (21).

Para los colaboradores de este estudio, asistir a los servicios de salud, en muchas ocasiones, implica quebrantar la independencia, la autonomía, la dignidad y la fortaleza, y entrar en conflicto con algunos valores religiosos. Estos resultados coinciden con estudios que han identificado cómo las creencias y los valores influyen en la búsqueda de ayuda $(15,20)$ y son constantes durante mucho tiempo e influyen en la toma de decisiones (22).

En este estudio las personas mayores con EC reconocen que los cuidados populares no sirven para curarlas y ven la necesidad de utilizar los servicios de salud y seguir a su manera los tratamientos y medicamentos prescritos. Esto hace visible la coherencia que construyen entre la comprensión de la situación de salud y las alternativas de búsqueda de atención.

Dentro de la ejecución del cuidado popular, los cuidados para mantener la salud se proporcionan de forma intermitente por parte de los colaboradores del estudio. Esta intermitencia puede ser explicada porque los síntomas no están presentes en el cuerpo y porque los cuidados populares también tienden a ser intermitentes. A pesar de que existe miedo ante las complicaciones de la enfermedad, las sensaciones del cuerpo evaden el inicio y continuidad del cuidado. Medel y González (23) encontraron, igualmente, que la preocupación y el miedo no motivan el inicio de conductas favorables para la salud. El sentimiento de miedo toma mayor importancia en los momentos de crisis, cuando pueden movilizarse cambios en las actitudes y mejor adherencia a los tratamientos (15).

Los cuidados populares implementados se interrumpen cuando sienten bienestar y aparece la creencia popular de "dejar descansar el cuerpo". Algunos autores encuentran que también la presencia de efectos adversos indeseables de los medicamentos se convierte en un factor desfavorecedor en el cumplimiento de los tratamientos $(24,25)$.

Por último, cabe resaltar que en los tres elementos de la trayectoria de cuidado popular de las personas mayores con EC, la familia aboga ante los servicios de salud o está pendiente para que las recomendaciones médicas se cumplan y determina qué se puede hacer y qué no respecto al cuidado durante las crisis y después de estas.

\section{Conclusiones}

Este estudio revela las similitudes de las trayectorias de cuidado popular para EC como la HTA, la diabetes y la EPOC. De forma específica, encontramos que las personas mayores con dichas enfermedades tienen pocos 
recursos de su cultura y de su experiencia para comprender su situación de salud, tomar decisiones y tratar su enfermedad. Así, estas personas buscan articular sus explicaciones de la EC y cuidados populares con las explicaciones y cuidados de los profesionales de la salud.

Las sensaciones del cuerpo son los elementos que llevan a la toma de decisiones y determinan la continuidad, la intermitencia o la suspensión de los cuidados populares, de los tratamientos médicos y de los medicamentos prescritos. "Dejar descansar el cuerpo" es una creencia popular muy arraigada en los colaboradores de este estudio, por lo cual es necesario recurrir a la negociación y a la reestructuración de estas creencias y prácticas de cuidado, con el fin de ayudar a mediar ante las barreras de acceso al sistema de salud.

La información presentada por los profesionales de la salud debe ser en un lenguaje común, culturalmente adaptada, útil, relevante y significativa. Se debe construir el concepto de EC, así como discutir las posibles complicaciones, pues ello permite que la persona mayor pueda preguntar y recomprender la enfermedad a partir de su experiencia (18). Los profesionales deben evaluar si la información se está asimilando y valorando adecuadamente y si los valores, las creencias y los conocimientos que poseen en relación con las medidas de prevención y control difieren de los suyos y por qué (26).

Tener en cuenta la perspectiva de las personas enfermas contribuye a planificar la atención médica y de servicios de salud, ya que ello aumenta la posibilidad de mejorar la calidad de los tratamientos y el autocuidado (16).

Este estudio confirma que las personas mayores con EC asisten a los servicios de salud en momentos de crisis, cuando peligra la vida, lo cual debe ser tenido en cuenta en el momento de la admisión. Al tenor, las enfermeras deberian brindar información adaptada que explique los beneficios de la hospitalización, para que sea vista no como sinónimo de muerte, sino como una posibilidad de vida. El conocimiento de las posibilidades evolutivas de su enfermedad por parte de los pacientes que acuden a urgencias por descompensación de su EC, va unido a una mayor implicación en las decisiones médicas y a un mayor deseo de información y participación (27).

\section{Agradecimientos}

A las personas mayores que participaron en este estudio y a los profesionales de salud de las unidades básicas de atención, por el apoyo brindado en la captación de los colaboradores y por el espacio brindado durante la asistencia a los controles médicos.

\section{Referencias}

1. Organización Mundial de la Salud (OMS). Temas de salud: envejecimiento [internet]. Ginebra: OMS; 2013 [acceso 2014 feb 7]. Disponible en: http://www.who.int/topics/ageing/es/index.html. 
2. Organización Mundial de la Salud (OMS). 10 datos sobre el envejecimiento de la población [internet]. Ginebra: OMS; 2013 [acceso 2013 jun 6]. Disponible en: http://www.who.int/features/factfiles/ageing/es/.

3. Organización Mundial de la Salud (OMS). Informe sobre la situación mundial de las enfermedades no trasmisibles 2010: resumen de orientación [internet]. Ginebra: OMS; 2011 [acceso 2013 dic 15]. Disponible en: http://www.who.int/nmh/publications/ncd_report_ summary_es.pdf.

4. Institute for Health Metrics and Evaluation. The global burden disease: generating evidence, guiding policy [internet]. Seattle: GBD Colombia; 2013. Disponible en: http://www.healthmetricsandevaluation.org.

5. Mercado Martínez FJ, Ramos Herrera IM, Valdez Curiel E. La perspectiva de enfermos crónicos sobre la atención médica en Guadalajara, México: un estudio cualitativo. Cad Saúde Pública. 2000;16(3):759-72.

6. Castellanos Soriano F, López L. Tejiendo explicaciones sobre vejez, discapacidad y pobreza en los cerros nororientales de Bogotá, Colombia. Investig Enferm. Imagen Desarr. 2011;13(2):27-47.

7. Castellanos Soriano F. Comprendiendo el cuidado de los ancianos en situación de discapacidad y pobreza [tesis de doctorado]. Bogotá: Universidad Nacional de Colombia, Facultad de Enfermería; 2009.

8. Leininger M. Transcultural Nursing. Concepts, theories, research and practices. 2nd ed. New York: McGraw Hill-College Custon Series; 1995.

9. Martínez M. Ciencia y arte en la metodología cualitativa. México: Trillas; 2004.

10. Guber R. La etnografia: método, campo y reflexividad. Bogotá: Norma; 2007.

11. Wolcott, H. Transforming qualitative data: description, analysis, and interpretation. Thousand Oaks, CA: Sage; 1994.

12. Spector RE. Las culturas de la salud. Madrid: Pearson Educación; 2003.

13. López A. O cuidar no contexto da adversidade: a história oral de idosos em uma comunidade colombiana [tese de doutorado em enfermagem]. São Paulo: Escola de Enfermagem da USP; 2003.

14. Peláez M, Vega E. Envejecimiento, pobreza y enfermedades crónicas en América Latina y el Caribe. Diabetes y Sociedad. 2005;51(4):30-3.

15. Machado LRC, Car MR. Dialectica do modo de vida de portadores de hipertensão arterial: objetivo e subjetivo. Rev Esc Enferm USP [internet]. 2007 [acceso 2011 ago 3];41(4):573-80. Disponible en: http://www.ee.usp.br/reeusp/

16. Mercado F, Hernández E. Las enfermedades crónicas desde la mirada de los enfermos y los profesionales de salud: un estudio cualitativo en México. Cad Saùde Pública. 2007;23(9):2178-86.

17. Campos-Navarro R, Torrez D, Arganis-Juárez E. Las representaciones del padecer en ancianos con enfermedades crónicas: un estudio en la Ciudad de México. Cad Saùde Pública. 2002;18(5):1271-9. 
18. Acosta González M, Debs Pérez G, de la Noval García R, Dueñas Herrera A. Conocimientos, creencias y prácticas en pacientes hipertensos, relacionados con su adherencia terapéutica. Rev Cubana Enfermer [internet]. 2005 Dic [citado 2011 jul 29]; 21(3): 1-1. Disponible en: http:/ / scielo.sld.cu/scielo.php?script=sci_arttext\&pid=S0864$03192005000300008 \& 1 n g=e s$.

19. López L, Alzate M, Velásquez V. Desarrollo de un modelo de cuidado cultural de la salud para personas ancianas con discapacidad en situación de pobreza en zonas urbano marginales de Bogotá y rurales de Bogotá y Cáqueza/Cundinamarca: 2005-2008. Bogotá: Universidad Nacional de Colombia, Facultad de Enfermería; 2008.

20. Lavielle P, Clark P, Martínez H, Mercado F. Ryan G. Conducta del enfermo ante el dolor crónico. Salud Pública Méx [internet]. 2008 Abr [citado 2011 nov 10];50(2):147-54. Disponible en: http:// www.scielo.org. $\mathrm{mx} /$ scielo.php?script=sci_arttext\&pid=S003636342008000200008\&lng=es.

21. Helman C. Cultura, saúde \& doença. $4^{\text {a }}$ ed. Porto Alegre (BR): Artmed; 2006.

22. Martin V, Roberto K. Assesing the stability of values and health care preferences of older adults: a long term comparison. J Gerontol Nurs. 2006;32(11):23-31.

23. Medel Romero BC, González Juárez L. Estilos de vida: experiencias con la adherencia al tratamiento no farmacológico en diabetes mellitus. Index Enferm [internet]. 2006 Jun [acceso 2013 may 16];15(5253):16-9. Disponible en: http://scielo.isciii.es/scielo.php?script=sci_ arttext\&pid=S1132-12962006000100004\&lng=es. http://dx.doi. org/10.4321/S1132-12962006000100004.

24. Corugedo Rodríguez MC, Martín Alfonso L, Bayarre Vea H. Adherencia terapéutica en pacientes con hipertensión arterial del Policlínico Universitario "Manuel Fajardo" en el municipio Cruces, Cienfuegos, 2009. Rev Cubana Med Gen Integr [internet] 2011 Dic [acceso 3 jun 2013];27(4):504-12. Disponible en: http://scielo.sld.cu/scielo. php?script=sci_arttext\&pid=S0864-2125201 1000400009\&lng=es.

25. Tosal Herrero B. El cuerpo como excusa: el diagnóstico de la fibromialgia en una consulta de reumatología. Index Enferm [internet]. 2008 [acceso 2013 mar 25];17(1). Disponible en: http:/ /www.indexf.com/index-enfermeria/v17n1/6600.php

26. Pires CGS, Mussi FC. Refletindo sobre pressupostos para o cuidar/ cuidado na educação em saùde da pessoa hipertensa. Rev Esc Enferm USP [internet]. 2009 [acceso 2013 mar 31];43(1):229-36. Disponible en: http://www.Ee.usp.br/reeusp/.

27. Antolin A, Sánchez M, Miro O. Evolución temporal en el conocimiento y el posicionamiento de los pacientes con enfermedades crónicas respecto al testamento vital. Gac Sanit [internet]. 2011 [acceso 2013 mar 31];25(5):412-8. Disponible en: http://scielo.isciii.es/pdf/gs / v25n5/original11.pdf. 
